\title{
DNA dC to dU-Editing Enzyme APOBEC-3A
}

National Cancer Institute

\section{Source}

National Cancer Institute. DNA dC to dU-Editing Enzyme APOBEC-3A. NCI Thesaurus. Code $C 117000$.

DNA dC->dU-editing enzyme APOBEC-3A (199 aa, $23 \mathrm{kDa}$ ) is encoded by the human APOBEC3A gene. This protein is involved in the modification of viral DNA. 